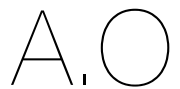

ARTIGO ORIGINAL

${ }^{1}$ Escola Superior de Hotelaria e Turismo do Estoril, Avenida Condes de

Barcelona, n. ${ }^{\circ} 808$ 2769-510 Estoril, Portugal

2 CITUR - Centro de Investigação,

Desenvolvimento e Inovação em Turismo - Pólo do Estoril, Avenida Condes de Barcelona, n..$^{\circ} 808$,

2769-510 Estoril, Portugal

${ }^{3}$ Faculdade de Ciências da Nutrição e Alimentação da Universidade do Porto, Rua Dr. Roberto Frias, $s / n$, 4200-465 Porto, Portugal

${ }^{4}$ GreenUPorto - Centro de Investigação em Produção Agroalimentar Sustentável, Campus de Vairão - Edifício de Ciências Agrárias (FCV2) Rua da Agrária, n. ${ }^{\circ} 747$,

4485-646 Vairão, Portugal

5 EPIUnit - Instituto de Saúde Pública da Universidade do Porto, Rua das Taipas, n. ${ }^{\circ} 135$ 4050-600 Porto, Portugal

${ }^{6}$ Escola Superior de Tecnologia da Saúde de Coimbra do Instituto Politécnico de Coimbra, Rua 5 de Outubro, s/n, 3046-854 Coimbra, Portuga

${ }^{7}$ CiTechCare - Center for Innovative Care and Health Technology,

Rua de Santo André, n.66-68, Campus 5 ,

Politécnico de Leiria,

2410-541 Leiria, Portugal

8 Universidade do Algarve Escola Superior de Saúde. Campus de Gambelas, 8005-139 Faro, Portugal

${ }^{9}$ LAQV-Requimte, Universidade do Porto, 4051-401 Porto, Portugal

‘Endereço para correspondência:

Ada Rocha

Faculdade de Ciências da Nutrição e Alimentação da Universidade do Porto, Rua Dr. Roberto Frias, $s / n$ 4200-465 Porto, Portuga adarocha@fcna.up.pt

Histórico do artigo

\section{OFERTA ALIMENTAR DE MENUS INFANTIS EM RESTAURANTES DE CENTROS COMERCIAIS PORTUGUESES: ESTUDO QUALITATIVO}

\author{
FOOD OFFER OF KIDS MENUS IN RESTAURANTS OF \\ PORTUGUESE SHOPPING CENTERS: QUALITATIVE STUDY
}

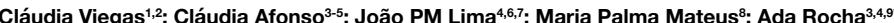

RESUMO

INTRODUÇÃo: O consumo de alimentos fora de casa aumentou e as famílias usam habitualmente os centros comerciais para realizar estas refeições. Os menus infantis disponíveis nos restaurantes são caracterizados por conterem alimentos com elevada densidade energética, ricos em gordura, gordura saturada, ácidos gordos trans e sódio e por uma oferta reduzida de hortícolas e fruta e de alimentos fornecedores de hidratos de carbono complexos, fontes alimentares de fibra e de vários micronutrientes. OBJETIVOS: Caracterizar a disponibilidade e a qualidade dos menus infantis nos restaurantes de centros comerciais em Portugal. METODOLOGIA: Foi realizado um estudo observacional de desenho transversal realizado em todos os centros comerciais de 6 cidades de Portugal. Para dar resposta aos objetivos propostos foi elaborada uma ferramenta de recolha de dados que incluiu três seções: A: Identificação do restaurante, B: Características do restaurante e C: Características do menu infantil.

RESULTADOS: Dos 184 restaurantes diferentes avaliados, apenas 44 disponibilizam menu infantil e, destes, 6 oferecem brindes associados ao mesmo. Os itens mais destacados nos menus são frango assado ou frito (25\%), hambúrgueres grelhados ou fritos (25\%), nuggets (18\%) e pizza (18\%). As batatas fritas são as opções mais frequentes de acompanhamento (41\%). Apenas 6 restaurantes disponibilizam sopa ou hortícolas adicionados à refeição. Embora a água estivesse disponível, refrigerantes e sumos de frutas açucarados também são opções disponibilizadas. A sobremesa doce é frequentemente disponibilizada no menu.

CONCLUSÕES: As opções do menu infantil analisadas oferecem alimentos nutricionalmente desadequados e baixa disponibilidade de hortícolas e frutas. Propostas de alterações aos alimentos oferecidos nos menus infantis revela-se uma estratégia importante na promoção de hábitos alimentares mais saudáveis nas crianças.

\section{PALAVRAS-CHAVE}

Avaliação qualitativa, Menus infantis, Restaurantes

ABSTRACT

INTRODUCTION: Out-of-home food consumption has increased and families eating out frequently use shopping centres. Kid's menus available at restaurants are characterized by contain foods with excessive energy, fat, saturated fat, -trans-fatty acids and salt and low vegetables, complex carbohydrate foods, fibre, and various micronutrients.

OBJECTIVES: Characterize the availability and quality of kid's menus in shopping centre restaurants in Portugal.

METHODOLOGY: An observational cross-sectional study was carried out in all shopping centres of 6 cities in Portugal. To answer the purposed aims a data collection tool was developed with three-section parts A: Identification of the restaurant, B: Characteristics of the restaurant and C: Characteristics of the kid's menu.

RESULTS: From 184 different restaurants evaluated, only 44 provide a kids menu and from these 6 restaurants offer gifts associated with it. The most frequently featured items on the menu are roast or fried chicken (25\%), hamburgers grilled or fried (25\%), nuggets (18\%) and pizza (18\%). Potato chips are the most frequent side dish options (41\%). Only 6 restaurants offered vegetable soup or vegetables added to the meal. Although the water was available, soft drinks and sugary fruit juices are also options. Sweet dessert is a common part of the menu.

CONCLUSIONS: Kids' menu options had poor quality with low availability of fruits and vegetables. Shifting foods offered to children in restaurants have the potential to improve diet quality, reduce excess energy intake and promote healthy eating habits.

\section{KEYWORDS}

Qualitative evaluation, Kids menu, Restaurant menus 


\section{INTRODUÇÃO}

O aumento da frequência do consumo alimentar fora de casa tem tido inúmeras consequências na saúde pública $(1,2)$. Diversos estudos têm documentado a associação entre o consumo alimentar fora de casa, particularmente em restaurantes de fast food e o consumo excessivo de energia, de alimentos/refeições ricas em gordura, gordura saturada $e$ sal, e o baixo consumo de leite, hortofrutícolas, alimentos fornecedores de hidratos de carbono complexos, fibra e diversos micronutrientes (1-11). Existe ainda evidência científica entre o consumo de fast food e a prevalência de sobrepeso e obesidade $(1,12,13)$.

Os restaurantes de fast food são um local de eleição para a realização de refeições por muitas famílias. De acordo com os dados publicados por Batada et al (2012), a maioria dos menus infantis caracteriza-se por ser de baixa densidade nutricional, com base em critérios como o teor de vitamina $\mathrm{A}$ e $\mathrm{C}$, cálcio, ferro, fibra, e ausência ou oferta reduzida de frutas, hortícolas e cereais $(8,14)$; um elevado valor energético e de gordura saturada, sódio e açúcar (1, 15-17).

O consumo alimentar em restaurantes tradicionais de rua tem vindo a diminuir, em consequência do aumento do número de centros comerciais, onde as praças de alimentação constituem fatores de diferenciação por concentrarem no mesmo local, funcionalidade, gastronomia e lazer (18).

O consumo alimentar fora de casa parece estar a aumentar, pois, segundo os Inquéritos aos Orçamentos das Famílias, os Portugueses gastaram em 2016 37,7\% do orçamento destinado à alimentação em consumo fora de casa, verificando-se um aumento de $17 \%$ e $22 \%$ em relação ao ano 2000 e 1990, respetivamente (19).

A maioria dos dados disponíveis têm-se focado nas tendências e a relação entre comer fora de casa e os padrões alimentares, sem terem sido avaliados a disponibilidade e o valor nutricional dos menus disponíveis nos restaurantes. Paralelamente, pouca investigação tem sido realizada sobre menus de restaurantes, especificamente dirigidos a crianças (20)

\section{OBJETIVOS}

Foi objetivo deste estudo caracterizar a disponibilidade de menus infantis em restaurantes de centros comerciais portugueses e avaliar qualitativamente a sua composição.

\section{METODOLOGIA}

Foi realizado um estudo observacional de desenho transversal. A recolha de dados ocorreu entre fevereiro e outubro de 2019 em todos os centros comerciais de seis cidades do litoral de Portugal continental: Porto, Gaia, Aveiro, Coimbra, Lisboa e Faro. A seleção das cidades alvo foi realizada por conveniência, e por serem considerados centros urbanos com uma densidade populacional relevante, representando as várias regiões de Portugal continental, de acordo com a NUTS II, e excluindo o Alentejo, devido ao reduzido número de centros comerciais nesta zona.

A ferramenta para recolha de dados foi desenvolvida pela equipa de investigadores, todos nutricionistas, sendo constituída por três secções: A: identificação do local e forma de obtenção da informação (local ou internet), B: características do estabelecimento de restauração e C: características do menu infantil. Relativamente às características do menu infantil foram recolhidas informações como o número de opções, itens que compõem o menu, a existência de opção vegetariana no menu infantil, presença de ofertas colecionáveis, informação nutricional e alergénios. Para todas as questões foram utilizadas respostas fechadas, dando como opções de itens do menu infantil: sopa, carne/pescado/ ovo, arroz/massa/batata, leguminosas, hortícolas, sobremesa doce e fruta e bebida. Adicionalmente, foram recolhidas fotografias dos menus infantis dos restaurantes analisados, de forma a permitir validar/ completar a informação recolhida.

Após elaboração do questionário, o mesmo foi validado pela metodologia de Delphi e posteriormente realizado o pré-teste num centro comercial de uma cidade não incluída no estudo. Garantiu-se que cada restaurante diferente foi visitado pelo menos duas vezes, para garantir a fiabilidade dos dados recolhidos.

A recolha dos dados foi feita a partir da informação que está publicamente disponível para os consumidores nos painéis informativos dos restautantes e, sempre que possível, validada confrontando com a informação que consta nos web sites de cada restaurante.

Os restaurantes analisados foram agrupados por tipologia, de acordo com o tipo de prato predominante: Asiático, Tradicional, Italiano, Hamburgueria e Outros

O tratamento estatístico foi realizado com recurso ao Microsoft Office Excel e posteriormente ao software R, versão 1.1.453 para Mac, sendo agrupados por zona geográfica e tipologia de restaurante.

\section{RESULTADOS}

Foi recolhida informação de 884 restaurantes. Cerca de 52\% dos restaurantes avaliados foram classificados como sendo do tipo fast food e 47,7\% como restaurantes tradicionais. As 884 recolhas efetuadas correspondem a 184 restaurantes diferentes sendo que apenas 44 (24\%) possuem menu infantil. Dos 139 estabelecimentos que não têm menu infantil, 5 disponibilizam aos pais a possibilidade de adquirir a mesma refeição do adulto, independentemente da opção selecionada, numa dose mais pequena e a um preço reduzido, havendo ainda um estabelecimento que na compra de duas refeições de adulto oferece a mesma refeição à criança.

Os restaurantes analisados foram classificados de acordo com a sua tipologia, observando-se um maior número de restaurantes do tipo tradicional, seguido de restaurante do tipo italiano (Tabela 1).

\section{Tabela 1}

Restaurantes com oferta de menu infantil de acordo com a tipologia de refeição servida

\begin{tabular}{|c|c|c|}
\hline TIPOLOGIA & $\mathbf{N}$ & $\%$ \\
\hline Asiático & 8 & 18 \\
\hline Tradicional & 13 & 30 \\
\hline Italiano & 10 & 25 \\
\hline Hamburgueria & 3 & 7 \\
\hline Outro & 10 & 23 \\
\hline Total & 44 & 100 \\
\hline
\end{tabular}

Os produtos oferecidos no menu que surgem com mais frequência são o frango assado ou frito (25\%), hambúrgueres grelhados ou fritos (25\%), nuggets (18\%), pizza (18\%), douradinhos (11\%) e esparguete à bolonhesa (11\%).

Como acompanhamento, a opção de batata frita é a mais frequente, surgindo em $41 \%$ dos restaurantes. De realçar que apenas seis dos 44 estabelecimentos oferecem sopa e seis oferecem hortícolas como complemento aos restantes componentes do prato, sendo em quatro sob a forma de salada de alface e dois cenoura ralada ou mini cenoura. Apenas quatro restaurantes apresentam opção vegetariana no menu infantil.

Quanto à bebida, 20 dos 44 estabelecimentos referem a água como uma das opções de bebida no menu infantil, mas a maioria oferece como alternativa uma gama variada de sumos e refrigerantes, até um máximo de oito variedades diferentes, onde se incluem refrigerantes, 
sumos de fruta industriais, néctares, colas, cola zero, chás, limonadas e sumos de fruta naturais. Num dos estabelecimentos é feita referência à disponibilidade, de cerveja e de café no menu infantil. Dos 44 estabelecimentos, três não incluem a bebida no preço do menu.

Observa-se que doze estabelecimentos oferecem sobremesa doce como parte do menu infantil, dos quais quatro apresentam também a fruta. Dos quatro estabelecimentos que apresentam fruta como sobremesa, apenas um a disponibiliza sem alternativa de sobremesa doce. A oferta de fruta limita-se apenas a uma variedade/opção de fruta (maçã, banana, boião de fruta) enquanto existem pelo menos duas opções doces, como gelado, gelatina e mousse de chocolate. Quanto às ofertas associadas aos menus infantis, seis dos estabelecimentos oferecem brindes associados ao menu infantil, na sua maioria brinquedos colecionáveis, existindo um estabelecimento cujo brinde é guloseimas à base de chocolate.

Em pelo menos três estabelecimentos a sobremesa tem um custo extra entre sessenta cêntimos e um euro. Em pelo menos dois o custo do menu permite escolher entre a sopa e a sobremesa.

Apenas dois estabelecimentos fazem referência aos hortícolas no título do menu, sendo que apenas $10 \%$ dos estabelecimentos possuem opção vegetariana.

Apenas 12,5\% disponibilizam informação referente aos alergénios no menu, nos restantes só por solicitação do cliente. Igual percentagem disponibiliza informação nutricional no menu.

Os restaurantes asiáticos oferecem como opções de menu infantil temakis, nigiris e sushi, e para além destes, buffets diversificados que incluem massas, fritos asiáticos e grelhados na chapa. No que diz respeito aos restaurantes italianos, para além das pizzas, surgem como opções massas com dois a três ingredientes à escolha, ou com molho carbonara e ainda lasanha. Nos restaurantes classificados como "tradicionais", as opões mais frequentes incluem cachorros, salsichas, almondegas, bifes de frango, lombo, bifanas, pregos, bitoque e panados. Em apenas dois restaurantes aparece a menção às opções de pescado, num dos quais esta é peixe frito e no outro pescada panada.

No que se refere aos principais métodos de confeção utilizados nas preparações destinadas aos menus infantis, observou-se que 53,6\% das opções são fritas, essencialmente distribuídos entre as opções de carne ou peixe (hambúrgueres, nuggets, douradinhos e bifes) e batata frita. Com percentagens inferiores surgem as opções cozidas com $12,9 \%$ (como as massas), mas que são normalmente acompanhadas de molhos à base de gordura, seguidos dos grelhados (10\%), dos assados $(7,1 \%)$, os crus $(9,3 \%)$ que correspondem às saladas e sushis e os estufados (5\%), que dizem respeito às bolonhesas.

Considerando apenas a componente carne ou pescado, o principal método de confeção é a fritura (69\%), seguido de grelhar (20\%). No que diz respeito aos acompanhamentos, o tipo de confeção mais utilizado é, novamente, a fritura (36\%), que diz respeito à batata frita, seguido da cozedura (27\%), normalmente utilizado na confeção de massa ou arroz.

\section{DISCUSSÃO DOS RESULTADOS}

Os resultados deste estudo revelam que a oferta alimentar caracteriza-se por carne vermelha e processada, açúcares simples, e inexistência ou baixa oferta de hortícolas, de frutas, de leguminosas e de cereais integrais. Diversos estudos demonstram que este tipo de oferta está associado a valores elevados de gordura geral, gordura saturada e trans, sal e pobreza em fibra e micronutrientes $(1,8,16)$. Estes resultados estão de acordo com o que tem sido observado noutros estudos, que reportam que a comida servida em restaurantes, particularmente a destinada a crianças, é de baixa densidade nutricional, com hambúrgueres, batatas fritas e sanduiches com recheio de alimentos ricos em gordura, como componentes centrais dos menus infantis em restaurantes e que a maioria dos restaurantes fornece pouca fruta, hortícolas, ou opções com baixo teor de gordura $(1,8,14-17,21,22)$.

A oferta alimentar, nos restaurantes avaliados, não cumpre as atuais recomendações alimentares, o que também já foi observado noutros estudos realizados nos EUA, nos quais foi calculado o grau de adesão ao Healthy Eating Index (HEI) em restaurantes de fast food populares, tendo sido verificado que os menus avaliados obtiveram uma pontuação inferior a 60, numa escala de 0 a 100, resultando numa oferta alimentar nutricionalmente pobre, em desacordo com as recomendações $(23,24)$. Um dos aspetos mais relevantes da má qualidade da oferta alimentar é 0 excesso de fritos, quer associados à componente carne/pescado, quer associados ao acompanhamento (batata frita), levando a que a oferta alimentar seja rica em gordura saturada e trans e, consequentemente, rica em energia, podendo resultar num valor energético acima das necessidades dos consumidores e em desacordo com as recomendações. Este é um aspeto também observado por McCrory, et al (2019) que estudaram os menus de fast food num período de 30 anos, tendo observado um aumento no tamanho das porções, das calorias e da quantidade de sal (25).

A oferta de um número muito superior de opções de carne versus pescado também constitui um dos problemas identificados, o qual poderá ser analisado do ponto de vista alimentar/nutricional, mas também ao nível do impacto ambiental.

O último Inquérito Alimentar Nacional e de Atividade Física refere que a população infantil apresenta um consumo de carne três vezes superior ao de pescado, sendo neste grupo que o consumo de pescado processado é mais elevado (26). Com exceção dos restaurantes de sushi, apenas dois estabelecimentos disponibilizam alternativas de peixe, e apenas quatro oferecem alternativas vegetarianas como fonte de proteína. A baixa oferta de pescado pode ser resultado de uma cultura instituída, na qual o consumo de carne é visto pelos pais, educadores e sociedade em geral como a fonte mais adequada e eficiente de proteína $(27,28)$. Nos últimos anos têm sido desenvolvidas campanhas de sensibilização por diversos organismos como a Direção-Geral da Saúde (29), a Associação Portuguesa de Nutrição (30), no sentido de promover alternativas de base vegetal (31-33), desmistificando a ideia da carne como fonte de proteína indispensável, facto que parece ainda não ter chegado a este tipo de oferta alimentar, onde a promoção dos produtos de origem animal permanece.

Aliado a este facto, a baixa oferta de produtos hortícolas é também um problema grave. O consumo de hortícolas na população infantil é baixo, representando menos de um terço do recomendado e dos consumidos apenas 36\% dizem respeito aos hortícolas no prato (26). A promoção do consumo de alimentos deste grupo deve ser realizada em diversos contextos, nomeadamente, em casa, e nas escolas, constituindo também a restauração um cenário de eleição ao nível da sua promoção (34). No entanto, o que se observa é que apenas seis dos 44 restaurantes disponibilizam hortícolas. $O$ consumo de produtos hortícolas é um dos aspetos com maior impacto sobre a saúde, referido pela Organização Mundial da Saúde como um dos fatores preventivos essenciais no desenvolvimento de Doenças Crónicas Não Transmissíveis (35). O seu baixo consumo, aliado ao elevado consumo de proteína de origem animal e de gordura saturada, provenientes sobretudo da carne, e de açúcar, aspetos também presentes nos menus avaliados, contribui para o desenvolvimento destas doenças, que constituem atualmente um problema de saúde mundial (36).

Apesar de quase metade dos estabelecimentos disponibilizar água no menu, todos oferecem alternativas ricas em açúcar, em número e 
variedade elevadas. Diversos autores, referem o consumo deste tipo de bebidas como uma fonte de açúcares livres que se associam a diversas patologias como obesidade, doença cardiovascular, diabetes tipo 2, entre outras (37-39). Uma das formas de limitar o consumo destas bebidas, seria criar um valor extra menu, para a sua aquisição, promovendo assim o consumo de água que estaria incluída no preço do menu.

A disponibilidade de café e cerveja nos menus infantis, apenas registada num dos estabelecimentos, é completamente desadequada e foi entendida pelos autores como um possível erro. Ainda assim, o facto do mesmo se manter revela falta de atenção e/ou conhecimento quer por parte dos responsáveis, quer por parte dos consumidores.

Apesar de apenas 12,5\% dos restaurantes avaliados apresentarem informação nutricional nos seus menus, estudos realizados em consumidores nos Estados Unidos da América mostraram que a maioria destes gostaria de visualizar o tamanho das porções e respetivas informações nutricionais $(40,41)$.

Contrariamente ao observado, face à oferta alimentar disponibilizada, os restaurantes poderão ser um ambiente importante para reduzir o consumo energético excessivo entre as crianças $(19,42)$, caso a oferta alimentar, nomeadamente ao nível dos menus infantis, seja alterada.

A literatura científica destaca vários caminhos potencialmente promissores para intervenções em restaurantes, nomeadamente através da associação à escolha de uma opção saudável de um brinde. Embora esses incentivos tradicionalmente promovam alimentos menos saudáveis em restaurantes (por exemplo, um brinquedo combinado com uma refeição infantil contendo entradas, batatas fritas e refrigerante), diversos estudos mostram que os incentivos também têm potencial para promover escolhas alimentares saudáveis (43-45). Uma segunda abordagem considera os pais e o papel que estes têm na escolha das refeições dos seus filhos $(46,47)$.

O encorajamento à compra mediante estratégias direcionadas para adultos como, por exemplo, o recurso a mensagens verbais $(48,49)$, sinalética/sinalização (50), e a inclusão de entradas saudáveis nos menus $(51,52)$, parece aumentar as vendas $(53)$, e pode promover padrões de pedidos mais saudáveis.

Vários estudos recentes apontam para uma maior aceitabilidade pelas crianças de opções mais saudáveis $(54,55)$, como a oferta de menus mais saudáveis em alguns restaurantes (54-56) e a incorporação de alimentos frescos e locais nos mesmos entre as principais tendências da indústria da restauração $(57,58)$.

Investigação adicional é necessária no sentido de testar estratégias adicionais que possam contribuir para a promoção da oferta de opções saudáveis para crianças nos menus dos restaurantes, ainda que o presente estudo, pela sua abrangência nacional, possa ser relevante para que a nível central se definam linhas de orientação direcionadas para a restauração, para que a oferta alimentar em idade infantil possa ser mais saudável.

\section{CONCLUSÕES}

Apenas $24 \%$ dos restaurantes avaliados possuem menu infantil e, globalmente, a qualidade da oferta alimentar destes é má. Caracteriza-se fundamentalmente pela oferta de opções à base de carne (essencialmente vermelha), cujo modo de confeção é maioritariamente a fritura, aplicável também aos acompanhamentos. A oferta de hortícolas no prato ou em sopa muito abaixo do desejável. As bebidas disponíveis são igualmente desadequadas, tendo em conta a oferta de refrigerantes e sumos, e até bebidas alcoólicas no menu infantil. A associação destas opções brinquedo colecionável ou guloseimas promovendo o consumo de opções menos adequadas. Esta estratégia podia ser utilizada para modelar escolhas mais saudáveis.

\section{AGRADECIMENTOS}

This research was supported by national funds through FCT - Foundation for Science and Technology within the scope of UIDB/05748/2020 and UIDP/05748/2020.

Os autores agradecem aos estudantes a participação na recolha de dados.

\section{REFERÊNCIAS BIBLIOGRÁFICAS}

1. Powell LM. Fast-Food and Full-service Restaurant Consumption among Children and Adolescents: Impact on Energy, Beverage and Nutrient Intake. JAMA Pediatr. 2013; 167(1):14-20.

2. Ferreira J. Restauração fora de casa em Portugal 2019: estado da arte. Institute of Marketing Research. 2019.

3. Foulds R. Kids restaurant meals worse than school dinners. The Food Magazine. 2004. 4. Lachat C, Roberfroid D HL, Van Camp J KP. Incorporating the catering sector in nutritions policies of WHO European Region: is there a good recipe? Public Health Nutr. 2009; 12(3):316-24. doi: 10.1017/S1368980008002176.

5. French SA SM, Neumark-Sztainer D, Fulkerson JA HP. Fast food restaurant use among adolescents: associations with nutrient intake, food choices and behavioral and psychosocial variables. Int J Obes Relat Metab Disord. 2001; 25:1823-33.

6. French SA HL JR. Fast food restaurant use among women in the Pound of Prevention study: dietary, behavioral and demographic correlates. Int J Obes Relat Metab Disord. 2000; 24:1353-9.

7. Soo J, Harris JL, Davison KK, Williams DR, Roberto CA. Changes in the nutritional quality of fast-food items marketed at restaurants, 2010 v . 2013. Public Health Nutr. 2018; 21(11):2117-2127. doi: 10.1017/S1368980018000629.

8. Garemo M, Naimi Al. Children's meals at restaurants in Abu Dhabi, United Arab Emirates, have poor nutritional quality. Mediterranean Journal of Nutrition and Metabolism. 2018; 11(12):1-8. http://doi.org/10.3233/MNM-17178.

9. Hay B. "Why do kids' menus always have chicken nuggets?": Children"s observations on the provision of food in hotels on family holidays. Hospitality \& Society. 2018; 8(1): 69-96. http://doi.org/10.1386/hosp.8.1.69_1.

10. Uechi K. Nutritional quality of meals offered to children ( kids ' meals ) at chain restaurants in Japan. Public Health Nutrition. 2018; 21(17):3101-10.

11. Todd, J. E., Mancino, L., \& Lin B-H. The impact of food away from home on adult diet quality. United States Department of Agriculture, Economic Research. 2010. disponivel em: http://www.ers.usda.gov/Publications/ERR90/ERR90.pdf.

12.Satia JA, Galanko JA S-RA. Eating at fast-food restaurants with dietary intake, Demographic Factors, psychosocial and behavioural North, among African Americans in North Carolina. Public Heal Nutr. 2004;7:1089-96.

13. Thompson OM BC, Resnicow $K$ et al. Food purchased away from home as a predictor of change in BMl z-score among girls. Int $\mathrm{J}$ Obes Relat Metab Disord. 2004;28:282-9.

14. Batada A, BrueningM ME, StoryM W. Poor nutrition on the menu: children's meals at America's top chain restaurants. Child Obes. 2012;8:251-4.

15. Moran AJ, Block JP, Goshev SG, Bleich SN, Roberto CA. Trends in Nutrient Content of Children ' s Menu Items. Am J Prev Med. 2016; 52(3):284-91.

16. Sliwa S, Anzman-frasca S, Lynskey V, Washburn K, Ed M, Economos C. Assessing the Availability of Healthier Children's Meals at Leading Quick-Service and Full-Service Restaurants. J Nutr Educ Behav. 2016; 48(4):242-249.e1.

17. Scourboutakos, M. J., Semnani-Azad, Z., \& L'Abbé MR. Added sugars in kids' meals from chain restaurants. Preventive Medicine Reports. 2016; 3(C):391-393.

18. Anzman-Frasca S, Folta SC, Glenn ME, Jones-mueller A, Lynskey VM, Patel AA, et al. Research Article Healthier Children 's Meals in Restaurants : An Exploratory Study to Inform Approaches That Are Acceptable Across Stakeholders. J Nutr Educ Behav. 2017; 49(4):285-295.e1. Available from: http://dx.doi.org/10.1016/j.jneb.2016.11.009. 19. Lopez N V, Folta SC, Glenn ME, Lynskey VM, Patel AA, Anzman-Frasca S. Promoting healthier children's meals at quick-service and full-service restaurants : Results from a pilot and feasibility study. Appetite. 2017 ;117:91-7. http://dx.doi.org/10.1016/j. appet.2017.06.015. 
20. Serrano EL, Jedda VB. Comparison of Fast-Food and Non-Fast-Food Children's Menu Items. J Nutr Educ Behav. 2009; 41(2):132-7. Available from: http://dx.doi. org/10.1016/j.jneb.2008.02.005.

21. Dammann K SC. Food-related attitudes and behaviors at home, school and restaurants: perspective from racially diverse, urban, low income 9-13 year old children in Minnesota. J Nutr Educ Behav. 2010; 42:389-97.

22. Edwards Z. Making a better choice for kids: what restaurants have to offer. J Nutr Educ Behav. 2010; 42(81).

23. Kirkpatrick SI, Reedy J, Kahle LL, Harris JL, Ohri-Vachaspati P, Krebs-Smith SM. Fast-food menu offerings vary in dietary quality, but are consistently poor. Public Health Nutr. 2013; 17(4):924-31. http://doi.org/10.1017/S1368980012005563.

24. Hearst MO, Harnack, LJ, Bauer KW, Earnest AA, French SA, Oakes JM. Nutritional Quality at Eight U.S. Fast-Food Chains. Am J Prev Med. 2013; 44(6):589-594. http:// doi.org/10.1016/j.amepre.2013.01.028.

25. McCrory MA et al. Fast-Food Offerings in the United States in 1986, 1991, and 2016 Show Large Increases in Food Variety, Portion Size, Dietary Energy, and Selected Micronutrients. J Acad Nutr Diet. 2019;119(6):923-33.

26. Lopes C, Torres D, Oliveira A, Severo M, Alarcão V, Guiomar S, Mota J, Teixeira P, Rodrigues S, Lobato L, Magalhães V, Correia D, Carvalho C, Pizarro A, Marques A, Vilela S, Oliveira L, Nicola P, Soares S, Ramos E. Inquérito Alimentar Nacional e de Atividade Física, IAN-AF 2015-2016: Relatório de resultados. Universidade do Porto, 2017. ISBN: 978-989-746-181-1. Disponivel em: www.ian-af.up.pt.

27. Schösler H, Boer J De. Towards more sustainable diets : Insights from the food philosophies of " gourmets " and their relevance for policy strategies. Appetite. 2018; 127:59-68. https://doi.org/10.1016/j.appet.2018.04.022.

28. Bryant C. A Survey of Consumer Perceptions of Plant-Based and Clean Meat in the USA, India, and China. Front. Sustain. Food Syst. 2019;3. https://doi.org/10.3389/ fsufs.2019.00011.

29. DGS. Programa Nacional de Promoção da Alimentação Saudável. 2019. Disponível em https://www.alimentacaosaudavel.dgs.pt/.

30. APN. Associação Portuguesa de Nutrição [Internet]. 2019. Disponível em https:// www.apn.org.pt/.

31. Rego $C$ et al. Alimentação saudável dos 0 aos 6 anos linhas de orientação para profissionais e educadores - Programa Nacional para a Promoção da Alimentação Saudável. Direção-Geral da Saúde. 2019.

32. Lobato L, Silva SG da, Cramês M, Santos CT, Graça P. Planeamento de refeições vegetarianas para crianças em restauração coletiva: princípios base. Programa Nacional para a Promoção da Alimentação Saudável. 2016.

33. Silva SCG, Pinho JP, Borges C, Santos CT, Santos A, Graça P. Linhas de orientação para uma alimentação vegetariana saudável. Programa Nacional para a Promoção da Alimentação Saudável. 2015.

34. WHO. European Food and Nutrition Action Plan 2015-2020. 2014. Disponíve em http://www.euro.who.int/__data/assets/pdf_file/0008/253727/64wd14e_ FoodNutAP_140426.pdf.

35. WHO. WHO Fruit and Vegetable Promotion Initiative. 2003.

36. WHO. Global action plan for the prevention and control of NCDs 20132020 [Internet]. 2013. Disponível: https://apps.who.int/iris/bitstream/ handle/10665/94384/9789241506236_eng.pdf?sequence=1.

37. Vos, M. B., Kaar, J. L., Welsh, J. A., Van Horn, L. V., Feig, D. I., Anderson, C. A. M. et al. Added Sugars and Cardiovascular Disease Risk in Children: A Scientific Statement From the American Heart Association. Circulation. 2016; 135(19). Disponível em http:// doi.org/10.1161/CIR.0000000000000439.

38. Jones, AC, Kirkpatrick, SI, Hammond D. Beverage consumption and energy intake among Canadians: analyses of 2004 and 2015 national dietary intake data. Nutrition Journal. 2019; 18(1):1-14. Available from: http://doi.org/10.1186/s12937-019-0488-5. 39. Gidding, SS, Dennison, BA, Birch, LL, Daniels, SR, Gillman, MW, Gilman, MW et al. Dietary recommendations for children and adolescents: a guide for practitioners: consensus statement from the American Heart Association. Circulation. 2005; 27;112(13):2061-75.

40. Colby SE, Johnson C ST, Gross K HK. Consumer attitudes and practices with nutrition labeling in grocery stores and on menus. J Nutr Educ Behav. 2009; 41(45). 41. Lando AM L-WJ. Helping consumers make more healthful food choices: consumer views on modifying food labels and providing point-of purchase nutrition information at quick-service restaurants. J Nutr Educ Behav. 2007;39:157-63.

42. Bevelander KE, Kwakernaak, Laset E, Zeinstra, G, Molleman G. Placing healthy kids meals on the restaurant menu by co-creation between chef-cooks and children. European Journal of Public Health. 2019; 29(4): 187-191. https://doi.org/10.1093/ eurpub/ckz187.191.

43. Cravener TL, Schlechter H, Loeb KL, Radnitz C, Schwartz M, Zucker N, et al. Feeding strategies derived from behavioral economics and psychology can increase vegetable intake in children as part of a home-based intervention: Results of a pilot study. J Acad Nutr Diet. 2015; 115(11):1798-807. http://dx.doi.org/10.1016/j. jand.2015.03.024.

44. McAlister AR, Cornwell TB. Collectible toys as marketing tools: Understanding preschool children's responses to foods paired with premiums. J Public Policy Mark. 2012; 31:195-205.

45. Morrill BA, Madden GJ, Wengreen, HJ, Fargo JD, Aguilar SS. A randomized controlled trial of the food dudes program: Tangible rewards are more effective than social rewards for increasing short-and long-term fruit and vegetable consumption. J Acad Nutr Diet. 2016; 116(4):618-29. http://dx.doi.org/10.1016/\%OAj.jand.2015.07.001.

46. Castro IA, Williams CB, Madanat H, Pickrel JL, Jun HJ, Zive M, et al. Food ordering for children in restaurants: Multiple sources of influence on decision making. Public Health Nutr. 2016; 19(13):2404-9. http://dx.doi.org/\%0A10.1017/ S1368980016001403.

47. Holmes, AS, Serrano EL, Machin JE, Duetsch T, Davis GC. Effect of different children's menu labeling designs on family purchases. Appetite. 2013; 62:198-202.

48. Schwartz J, Riis J, Elbel B, Ariely D. Inviting consumers to downsize fast-food portions significantly reduces calorie consumption. Health Aff. 2012; 31:399-407. http://dx.doi.org/10.1377/hlthaff.2011.0224.

49. Kleef E, Broek O, Trijp H. Exploiting the spur of the moment to enhance healthy consumption: Verbal prompting to increase fruit choices in a selfservice restaurant. Appl Psychol Heal Well-Being. 2015; 7:149-66.

50. Nothwehr FK, Snetselaar L, Dawson J, Schultz U. Promoting healthy choices in non-chain restaurants: Effects of a simple cue to customers. Health Promot Pract. 2013; 14(132-138).

51. Papies EK, Potjes I, Keesman M, Schwinghammer S, Koningsbruggen GM Van. Using health primes to reduce unhealthy snack purchases among overweight consumers in a grocery store. Int J Obes. 2013;38(4):597-602. http://dx.doi. org/10.1038/ijo.2013.136.

52. Stutts MA, Zank GM, Smith KH, Williams SA. Nutrition information and children's fast food menu choices. J Consum Aff. 2011; 45(1):52-86.

53. Ebster, C., Wagner, U., \& Valis S. The effectiveness of verbal prompts on sales. J Retail Consum Serv. 2006;13:169-76.

54. Anzman-Frasca S, Dawes F SS, Al E. Healthier side dishes at restaurants: an analysis of children's perspectives, menu content, and energy impacts. Int $\mathrm{J}$ Behav Nutr Phys Act. 2014; 11(81).

55. Anzman-Frasca S MM, Sliwa S et al. Changes in children's meal orders following healthy menu modifications at a regional US restaurant chain. Obes (Silver Spring). 2015; 23:1055-62.

56. Hearst MO, Harnack LJ BK, Earnest AA, French SA M, J. O. Nutritional quality at eight U.S. fast-food chains: 14-year trends. Am J Prev Med. 2013; 44:589-94.

57. What 's hot: 2019 Culinary forecast. National Restaurant Association. 2019. Disponível em https://restaurant.org/Downloads/PDFs/Research/WhatsHot/ WhatsHotFinal2019.

58. What's hot: 2015 Culinary forecast. 2015. National Restaurant Association. 2015. Disponível em https://www.restaurant.org/Downloads/PDFs/Research/WhatsHot/ WhatsHot2015-Results. 